\title{
OSCILLATION AND PERIOD DOUBLING IN TCP/RED SYSTEM: ANALYSIS AND VERIFICATION
}

\author{
XI CHEN, SIU-CHUNG WONG, CHI K. TSE* \\ and FRANCIS C. M. LAU \\ Department of Electronic and Information Engineering, \\ Hong Kong Polytechnic University, \\ Hong Kong, P. R. China \\ *encktse@polyu.edu.hk
}

Received April 20, 2007; Revised May 23, 2007

\begin{abstract}
It has been known that a bottleneck RED (Random Early Detection) gateway can become oscillatory when regulating multiple identical TCP (Transmission Control Protocol) flows. However, a systematic explanation of such oscillatory behavior is not available. In this paper, we first use the fluid-flow model to derive the system characteristic frequency, and then compare with the frequencies of the RED queue length waveforms observed from "ns-2" simulations. The "ns-2" simulator is the only viable simulation tool accepted by industry for verification purposes. Analysis of the TCP source frequency distribution reveals the occurrence of period doubling when the system enters the instability region as the filter resolution varies. Since random events and a large number of TCP flows are involved in the process of generating the average system dynamics, a statistical viewpoint is taken in the analysis. Our results reflect the true system behavior as they are based on data from "ns-2" simulations rather than numerical simulations of analytical models. The physical mechanism of oscillation is explained in terms of the difference in the TCP source frequency and the TCP-RED system characteristic frequency.
\end{abstract}

Keywords: TCP/IP protocol; RED gateway; dynamics; period doubling; bifurcation.

\section{Introduction}

Conventional media of information exchange and distribution have gradually given way to the more efficient, rapid, economical and globalized infrastructure of information exchange network - the Internet. This huge infrastructure of information exchange/transfer consists of many interconnected gateways and uses the TCP/IP protocol suite to provide reliable services of transporting information from one place to another as well as to ensure an even sharing of information flow among different connections. In practice, there are bottleneck links which are required to serve a large number of TCP flows. The overall network utilization is therefore limited by the ability of the bottleneck links in effectively sharing their bandwidths among all the flows connected to them. The job of a gateway is thus to regulate the flows in a bottleneck link so that its bandwidth shares evenly among the many flows connected to it.

Normally, two endpoints of a TCP connection communicate using a so-called window-based flow, which can be described as follows. In the sender, a stream of data is partitioned into a sequence of windows of packets. The packets in a window are sent in one batch of size $w$. When the packet is successfully received, the receiver returns an acknowledgment packet. After receiving the first packet acknowledgment from the last window, the sender 
then starts sending packets in the next window, and so on. The time duration between the sending of a packet and the receiving of the acknowledgment of the same packet is defined as the round-trip-time (RTT). Thus, a total of $w$ packets is sent for each RTT. In practice, the window size $w$ is adaptively adjusted under a TCP algorithm to reach its optimal share of throughput according to the network conditions, and the TCP communication will eventually reach its steady state as a self-regulated RTT clocked system.

A few TCP algorithms have been proposed for dynamically controlling the window size according to the network conditions, e.g. Tahoe [Jacobson, 1988], Reno [Jacobson, 1990] and Vegas [Brakmo \& Peterson, 1995] or their variants. Tahoe and Reno employ the Additive Increase Multiplicative Decrease (AIMD) algorithm for congestion avoidance. Vegas uses a more proactive approach for controlling window size. At present, Reno is the most widely used TCP algorithm. Our focus here is the AIMD congestion avoidance algorithm based on Tahoe and Reno implementations.
The AIMD congestion avoidance algorithm can be described as follows. The window size $w$ increases linearly by $1 / w$ for each acknowledgment returned. This is known as Additive Increase. Thus, after $w$ packets (a window of packets) are transmitted, $w$ will become $w+1$ within one RTT. A congestion is indicated either by receiving three duplicated acknowledgments of a particular packet sent or by an Explicit Congestion Notification (ECN) header information injected from a gateway and forwarded in the acknowledgment by the receiver. Under such a circumstance, $w$ is abruptly reduced to $w / 2$. This is called Multiplicative Decrease. After that, the additive increase algorithm resumes.

The aim of the congestion avoidance algorithm in a TCP sender is to optimize its fair share of bandwidth in the links connecting the TCP endpoints. At least one of the links along the path of the communication channel becomes the bottleneck link. In this bottleneck link, an active queue management (AQM) gateway regulates the flow so as to maximize capacity. The simplest AQM gateway is the drop tail gateway, which drops the incoming

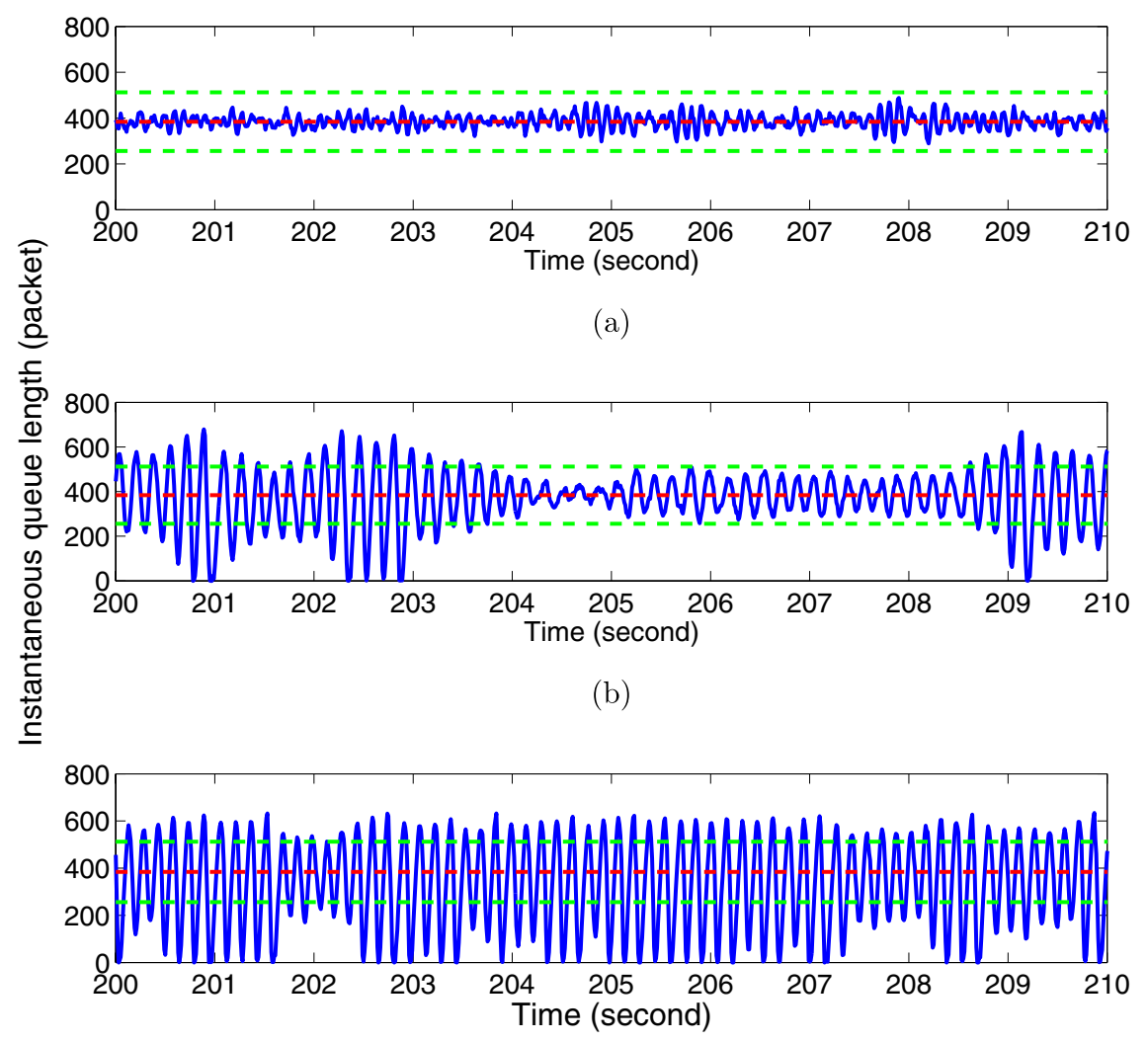

(c)

Fig. 1. Simulated queue length waveforms of TCP-RED using the $n s-2$ simulator for different values of filter resolution $\alpha$. (a) $\alpha=0.1$, (b) $\alpha=0.0005$ and (c) $\alpha=0.0008$ for 170 TCP connections. Each connection shares a fixed bandwidth of $1.5 \mathrm{Mb} / \mathrm{s}$ in the bottleneck link. 
packets when its queue buffer becomes full. However, this drop tail gateway may suffer from poor communication with the TCP senders since a large amount of packets can be dropped as the senders may keep sending while the gateway may keep dropping packets until a retransmission time-out breaks the loop. Random Early Detection (RED) [Floyd \& Jacobson, 1997] gateway was introduced to provide an early indication to the TCP senders by marking (or dropping) packets in advance and well before the buffer becomes full. The aim is to prevent large amount of packets from being dropped. However, it has been found that RED may have instability and oscillatory problems [Misra et al., 2000; Firoiu \& Borden, 2000; Floyd et al., 2001; Ranjan \& Abed, 2002; Chen et al., 2004], which degrade the transmission performance. We have performed simulations using the industry standard simulator $n s-2$ [DARPA, 2007]. ${ }^{1}$ As shown in Fig. 1, ns-2 simulations have clearly illustrated the occurrence of oscillation at frequencies related to the TCP sending rate as well as intermittent behaviors.

In this paper, we will analyze the TCP-RED system. One of our aims is to give a physical explanation on the mechanism of instabilities in this system. The calculation of the characteristic frequency of the TCP-RED system using the fluid-flow model will be given in Sec. 2. In Secs. 3 and 4, the single characteristic frequency of the fluid-flow model will be compared with the statistical distribution of frequencies obtained from $n s$ - 2 simulations of the RED queue length. Instability in the form of period doubling of the aggregated TCP source frequencies will be illustrated in Sec. 4.1. Physical explanation for the observed oscillation will be given in Sec. 4.2. Finally we conclude in Sec. 5.

\section{Overview of Operation}

Figure 2 shows a system of $N$ TCP flows that pass through a bottleneck RED gateway [Firoiu \& Borden, 2000; Ranjan \& Abed, 2002; Chen et al.,

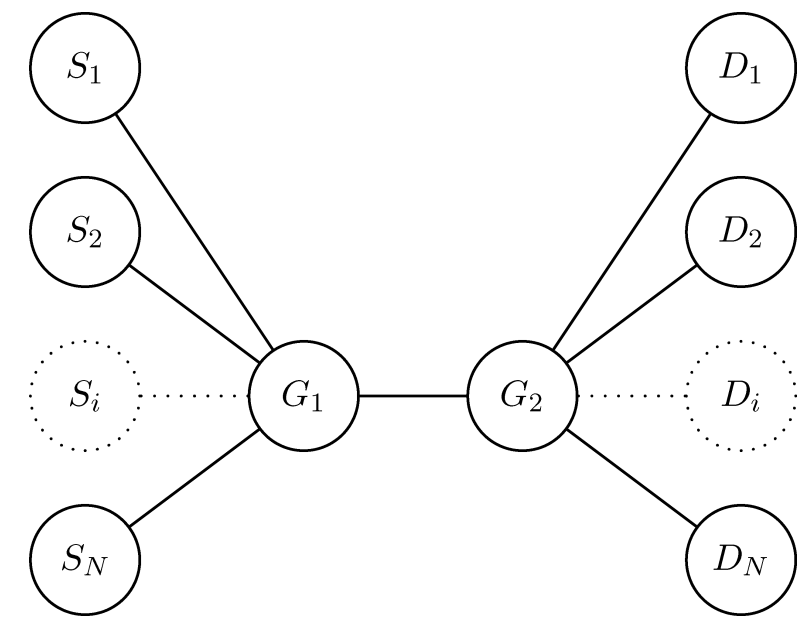

Fig. 2. A system of $N$ TCP flows, from $S_{i}$ to $D_{i}$, where $i=1,2, \ldots, N$, passing through a common bottleneck link between $G_{1}$ and $G_{2}$.

2004]. It is assumed that the system is operating in its desired stable condition [Floyd \& Jacobson, 1997]. Also, the system uses an AIMD algorithm with ECN capability, as described earlier. The $N$ long-live TCP connections are essentially controlled by the ECN markings of the RED gateway. The ECN markings are controlled by the RED gateway to maintain an even distribution among the $N$ TCP flows, making them share evenly the bandwidth of the bottleneck link.

\subsection{Fluid-flow model}

The dynamics of the queue length and window size can be described by a fluid model [Misra et al., 2000], which is described as follows:

$$
\begin{aligned}
\frac{d w(t)}{d t} & =\frac{1}{r(t)}-\frac{w(t)}{2} \frac{w(t-r(t))}{r(t-r(t))} p(t-r(t)) \\
\frac{d q(t)}{d t} & =N \frac{w(t)}{r(t)}-C \\
\frac{d x(t)}{d t} & =C \ln (1-\alpha)(x(t)-q(t)),
\end{aligned}
$$

$$
p_{b}(t)= \begin{cases}0 & \text { for } 0 \leq x(t)<X_{\min }, \\ \frac{x(t)-X_{\min }}{X_{\max }-X_{\min }} p_{\max } & \text { for } X_{\min } \leq x(t) \leq X_{\max }, \\ p_{\max }+\frac{1-p_{\max }}{X_{\max }}\left(x(t)-X_{\max }\right) & \text { for } X_{\max }<x(t) \leq 2 X_{\max }, \\ 1 & \text { for } 2 X_{\max } \leq x(t) \leq B\end{cases}
$$

\footnotetext{
${ }^{1}$ The $n s$ - 2 simulator is regarded as a viable simulation tool for the study of the behavior of TCP networks, and is the only acceptable verification tool that reflects the true behavior of TCP flow dynamics.
} 


$$
\begin{aligned}
& p(t)=\kappa p_{b}(t) \\
& r(t)=\frac{q(t)}{C}+R_{0}
\end{aligned}
$$

where $w(t)$ is the averaged instantaneous window size (in packets) of the TCP sources; $r(t)$ is the round trip time; $q(t)$ is the averaged instantaneous queue length (in packets); $x(t)$ is the filtered queue length after removal of short bursts; $p(t)$ is the marking probability; $\alpha$ is the filter resolution $(0<$ $\alpha<1) ; \kappa$ is a proportionality constant dependent upon the detailed implementation of the RED algorithm; $X_{\max }$ is the maximum threshold of $x(t) ; X_{\min }$ is the minimum threshold of $x(t) ; p_{\max }$ is the maximum threshold of $p(t) ; R_{0}$ is the propagation delay; $C$ is the bottleneck bandwidth; and $B$ is the maximum physical queue length.

Basically, Eqs. (1)-(6) describe a closed-loop system, whose operation can be explained as follows. First, $w$ is the averaged (expected) instantaneous window size (in packets) of the TCP sources, and it is controlled by the congestion avoidance algorithm. Basically $w$ increases by one for each round-trip-time $r(t)$ and decreases by $w(t) / 2$ for each ECN marking received. The round-trip-time $r(t)$ is the sum of the queue delay $q(t) / C$ and the propagation delay $R_{0}$. The ECN marking was sent previously from the RED gateway at a time $r(t)$ ago and is therefore proportional to the product of its share of flow rate $w(t-r(t)) / r(t-r(t))$ and the marking probability $p(t-r(t))$. Also, $q(t)$ is the averaged instantaneous queue length whose rate of change is proportional to the total number of TCP flows minus the bottleneck queue capacity (packet/sec). Also, $x$ is the filtered output from $q$, after short bursts of flow have been removed. The probability $p(t)$ is given by (4) and (5). The proportionality constant $\kappa$ in (5) depends on the implementation details of the RED algorithm.

In the following subsections, we will find the characteristic oscillatory frequencies for the system. If the characteristic oscillation is being "encouraged" by the closed loop, we will observe oscillatory waveforms dominated at the characteristic frequency, as shown in Fig. 1.

The model has been analyzed using small-signal linearization and perturbation [Hollot et al., 2001; Hollot \& Chait, 2001; Park et al., 2004; Chen et al., 2007] for the calculation of instability boundaries. Our aim here is to study the detailed dynamics of interactions among individual TCP sources and their aggregated dynamics interacting with the same bottleneck link (controlled by the RED gateway), and therefore to explain the detailed physical mechanism of the oscillations of the RED queue length.

\subsection{Steady-state solution}

We consider the TCP-RED system as a whole and assume that the TCP-RED system is stable under a certain control algorithm. In the steady state, the system converges to an equilibrium point $\left(q_{o}, r_{o}, w_{o}, p_{o}\right)$, which is given as

$$
\left\{\begin{array}{l}
q_{o}=\frac{X_{\max }+X_{\min }}{\Phi} \\
r_{o}=\frac{q_{o}}{C}+R_{o} \\
w_{o}=\frac{C r_{o}}{N} \\
p_{o}=\kappa p_{\max } \frac{1+(1-\Phi) \frac{X_{\min }}{X_{\max }}}{\Phi\left(1-\frac{X_{\min }}{X_{\max }}\right)}=2\left(\frac{N}{C r_{o}}\right)^{2},
\end{array}\right.
$$

where $q_{o}$ is the target queue length, the constant $\Phi$ is usually chosen to be equal to 2 to achieve maximum symmetrical swing of the queue length and, thereby, minimize the queue length saturation. The steady-state probability $p_{o}$ is a function of $q_{o}$.

\subsection{Small-signal analysis}

To facilitate analysis, one may linearize (1), (2) and (5) at the equilibrium point and obtain

$$
\left\{\begin{aligned}
\delta \dot{w}(t)= & \frac{-N}{r_{o}^{2} C}\left[\delta w(t)+\delta w\left(t-r_{o}\right)\right] \\
& -\frac{N}{r_{o}^{2} C}\left[\delta q(t)+\delta q\left(t-r_{o}\right)\right] \\
& -\frac{r_{o} C^{2}}{2 N} \delta p\left(t-r_{o}\right) \\
\delta \dot{q}(t)= & \frac{N}{r_{o}} \delta w(t)-\frac{1}{r_{o}} \delta q(t) \\
\delta \dot{p}(t)= & C \ln (1-\alpha)(\delta p(t)-\beta \delta q(t)),
\end{aligned}\right.
$$

where

$$
\left\{\begin{array}{l}
\delta w=w-w_{o} \\
\delta q=q-q_{o} \\
\delta p=p-p_{o}
\end{array}\right.
$$


are the perturbed variables at the equilibrium point and $\beta=\kappa p_{\max } /\left(X_{\max }-X_{\min }\right)$. Thus, (8) can be rewritten as

$$
\begin{aligned}
\delta \ddot{p}(t) & +\left(-\alpha_{1} C+\frac{1}{r_{0}}+\frac{N}{C r_{0}^{2}}\right) \delta \ddot{p}(t) \\
& +\left(-\frac{\alpha_{1} C}{r_{0}}-\frac{N \alpha_{1}}{r_{0}^{2}}+\frac{2 N}{C r_{0}^{3}}\right) \delta \dot{p}(t) \\
& -\frac{2 N \alpha_{1}}{r_{0}^{3}} \delta p(t)+\frac{N}{C r_{0}^{2}} \delta \ddot{p}\left(t-r_{0}\right) \\
& -\frac{N \alpha_{1}}{r_{0}^{2}} \delta \dot{p}\left(t-r_{0}\right)-\frac{C^{3} \alpha_{1} \beta}{2 N} \delta p\left(t-r_{0}\right)=0
\end{aligned}
$$

where $\alpha_{1}=\ln (1-\alpha)$. The above equation can be written as

$$
\begin{aligned}
\delta \dddot{p}(t) & +a_{1} \delta \ddot{p}(t)+a_{2} \delta \dot{p}(t)+a_{3} \delta p(t)+b_{1} \delta \ddot{p}(t-r(t)) \\
& +b_{2} \delta \dot{p}(t-r(t))+b_{3} \delta p(t-r(t))=0
\end{aligned}
$$

where

$$
\left\{\begin{array}{l}
a_{1}=\left(-\alpha_{1}+\frac{1}{K}+\frac{N}{K^{2}}\right) C \\
a_{2}=\left(-\frac{\alpha_{1}}{K}-\frac{N \alpha_{1}}{K^{2}}+\frac{2 N}{K^{3}}\right) C^{2} \\
a_{3}=-\frac{2 N \alpha_{1}}{K^{3}} C^{3} \\
b_{1}=\frac{N}{K^{2}} C \\
b_{2}=-\frac{N \alpha_{1}}{K^{2}} C^{2} \\
b_{3}=-\frac{\alpha_{1} \beta}{2 N} C^{3}
\end{array}\right.
$$

and $K=C r_{0}$. We will use (11) in the next subsection to determine the characteristic frequency of oscillation of the TCP-RED system.

\subsection{Finding the characteristic frequency}

To find the characteristic frequency, we consider the Fourier expansions of all the system variables. For instance, $\delta p$ is written in the form of $\delta p=A e^{j \theta(t)}$ where $j=\sqrt{-1}, \theta(t)=\omega t+\phi$, with $\omega$ and $\phi$ denoting the frequency and phase, respectively. Thus, we can write

$$
\left\{\begin{array}{l}
\delta p(t)=A e^{j(\omega t+\phi)} \\
\delta \dot{p}(t)=j A \omega e^{j(\omega t+\phi)} \\
\delta \ddot{p}(t)=-A \omega^{2} e^{j(\omega t+\phi)} \\
\delta \ddot{p}(t)=-j A \omega^{3} e^{j(\omega t+\phi)} \\
\delta p\left(t-r_{0}\right)=A e^{j\left(\omega\left(t-r_{0}\right)+\phi\right)} \\
\delta \dot{p}\left(t-r_{0}\right)=j A \omega e^{j\left(\omega\left(t-r_{0}\right)+\phi\right)} \\
\delta \ddot{p}\left(t-r_{0}\right)=-A \omega^{2} e^{j\left(\omega\left(t-r_{0}\right)+\phi\right)} .
\end{array}\right.
$$

Substituting (13) into (11), we obtain:

$$
\begin{aligned}
& -j \omega^{3}-a_{1} \omega^{2}+a_{2} j \omega+a_{3} \\
& \quad-b_{1} \omega^{2}\left[\cos \left(\omega r_{0}\right)-j \sin \left(\omega r_{0}\right)\right] \\
& \quad+j b_{2} \omega\left[\cos \left(\omega r_{0}\right)-j \sin \left(\omega r_{0}\right)\right] \\
& \quad+b_{3}\left[\cos \left(\omega r_{0}\right)-j \sin \left(\omega r_{0}\right)\right]=0 .
\end{aligned}
$$

Separating real and imaginary parts, we get

$$
\left\{\begin{aligned}
-a_{1} \omega^{2}+a_{3}= & \left(b_{1} \omega^{2}-b_{3}\right) \cos \left(\omega r_{0}\right) \\
& -b_{2} \omega \sin \left(\omega r_{0}\right) \\
\omega^{3}-a_{2} \omega= & \left(b_{1} \omega^{2}-b_{3}\right) \sin (\omega) \\
& +b_{2} \omega \cos \left(\omega r_{0}\right) .
\end{aligned}\right.
$$

Hence, the characteristic frequency can be obtained by solving the positive real roots of the following equation:

$$
\begin{aligned}
\omega^{6}+ & \left(a_{1}^{2}-2 a_{2}-b_{1}^{2}\right) \omega^{4}+\left(a_{2}^{2}-2 a_{1} a_{3}-b_{2}^{2}\right. \\
& \left.+2 b_{1} b_{3}\right) \omega^{2}+\left(a_{3}^{2}-b_{3}^{2}\right)=0 .
\end{aligned}
$$

\section{Actual Steady-State Waveforms of TCP Sources}

In the foregoing section, we have derived the characteristic oscillation frequency from the fluid-flow

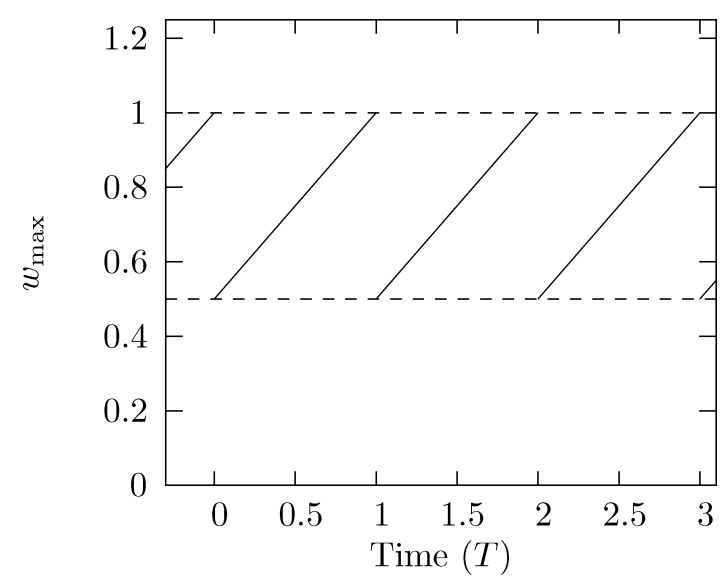

Fig. 3. Ideal steady-state waveform of TCP sender's window size. 
model analytically. In doing so, the individual dynamics of the TCP sources have been averaged to give the mean dynamics without considering the statistical distribution of individual system variables arising out of the random process of the TCPRED system. In this section, we will compare the waveforms of the TCP source window size obtained from $n s$ - 2 simulations with those obtained from the linearized fluid-flow model.

In the steady state, a TCP source maintains its share of bandwidth using the congestion avoidance algorithm. Figure 3 shows an ideal TCP window steady-state waveform. It has been shown [Mathis et al., 1997] that the TCP sources switch their
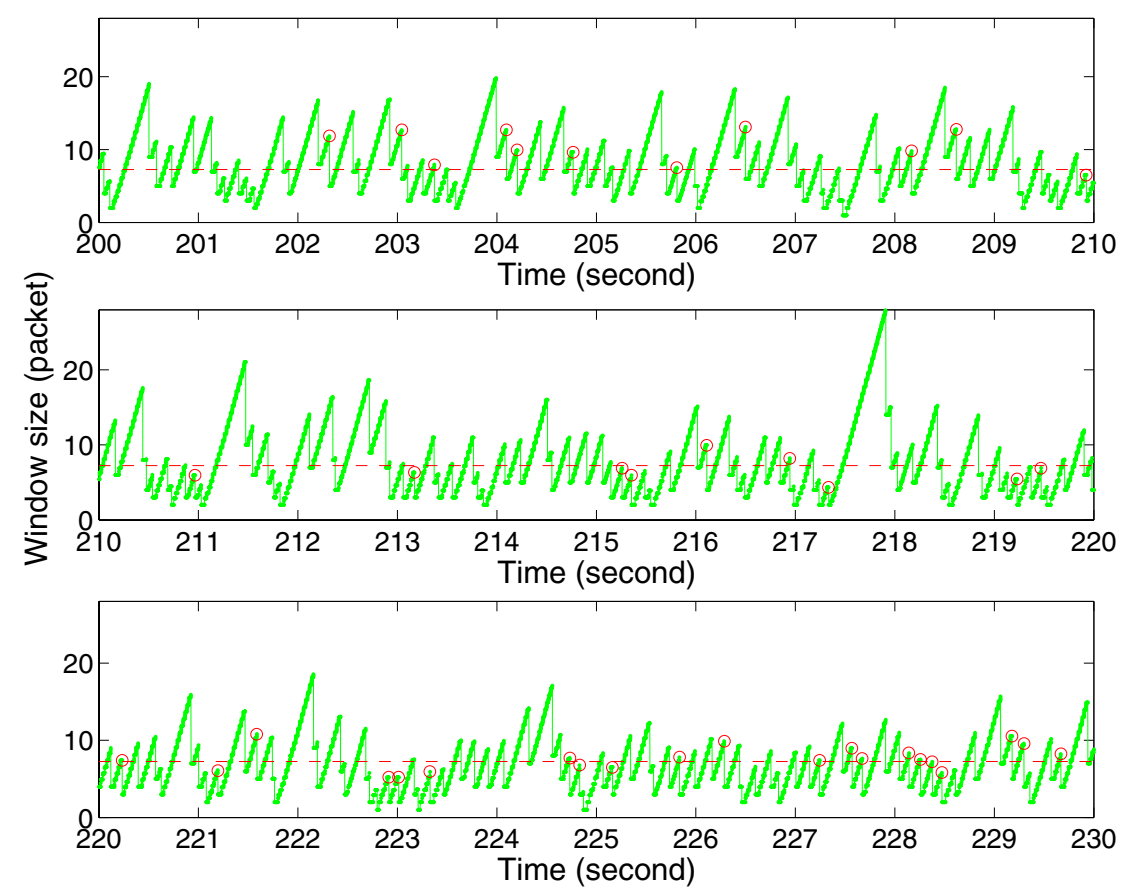

Fig. 4. Waveform of TCP source window size of a connection at filter resolution $\alpha=0.1$.
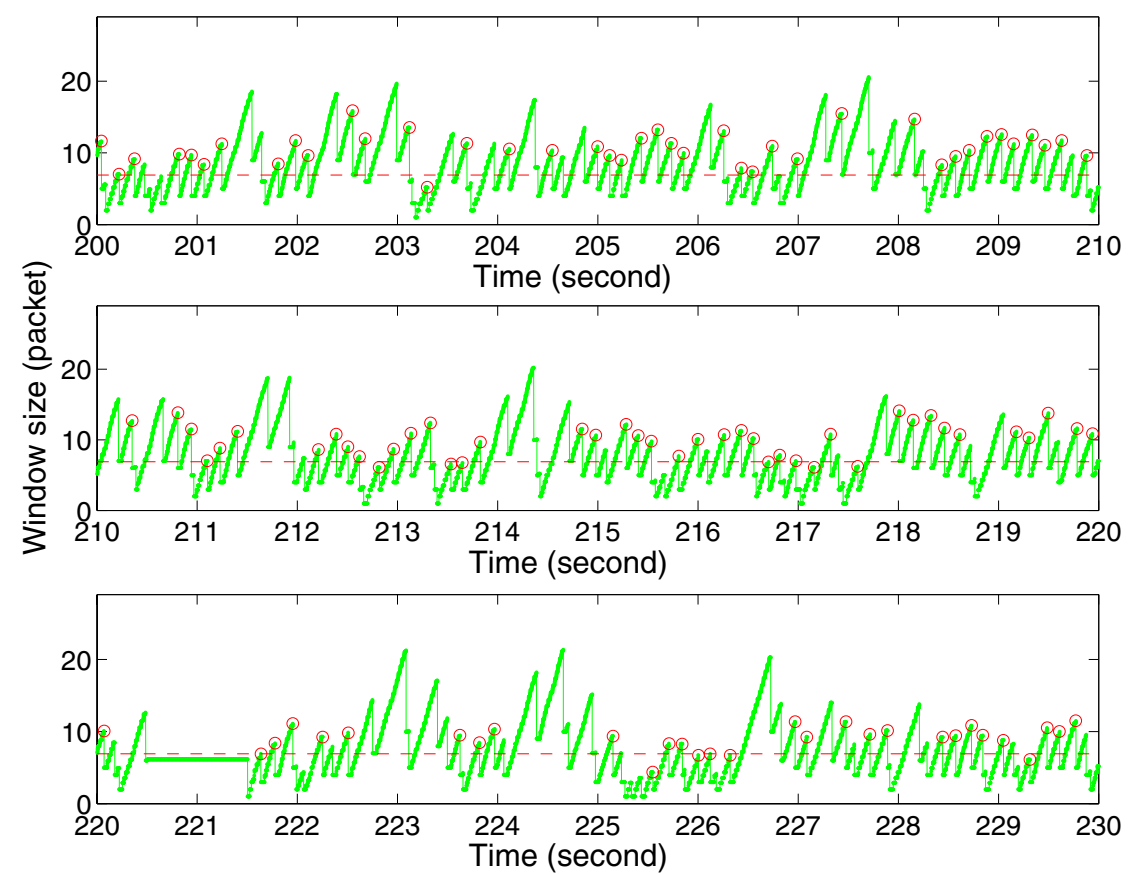

Fig. 5. Waveform of TCP source window size of a connection at filter resolution $\alpha=0.001$. 
sending rates at a period of $T$ given by

$$
T=\frac{w_{\max } r_{o}}{2}
$$

where $w_{\max }$ is the maximum of $w(t)$ and $r_{o}$ is the average RTT, within a TCP sender period.

We observe from Fig. 1 that $n s$ - 2 simulations reveal a characteristic frequency of the queue length waveform which is related to the statistical distribution of $1 / T$. Two close-up views of a randomly chosen TCP source window waveform are shown in Figs. 4 and 5, which are clearly different from the idealized steady-state waveform at any portion of time durations.

In all these cases, we observe that while the long-term average flow rate and window period are maintained at $3 w_{\max } / 4 r_{o}$ and $T$, respectively, the instantaneous average flow rate and window period deviate significantly from these long-term average values. In the next section we will use a statistical method to compare the dynamics of waveforms calculated from the fluid-flow model and the actual waveforms simulated using the $n s-2$ simulator.

\section{Comparing Fluid-Flow Model Calculations and $n s-2$ Simulations}

In studying the dynamics of the interaction between the aggregate flow of all the TCP sources and the
RED gateway, we choose an experimental setup which uses the RED filter resolution $\alpha$ as a single varying parameter. As $\alpha$ is varied, the system crosses the boundary of stability, revealing the dynamical features of TCP sources and the whole TCP-RED system.

Figure 6 shows the variation of the characteristic frequency as $\alpha$ is changed with other system parameters set as shown in Table 1. The oscillation frequencies obtained from the two methods agree perfectly. There are differences in interpreting the two systems. For the linearized fluid-flow system, the characteristic frequency, if exists, will be the only frequency observed. The oscillation at the characteristic frequency will normally decay to zero amplitude with a time constant which increases as the system approaches the instability boundary, as shown in Fig. 7(a). This corresponds to stable operation. Moreover, if the oscillation is sustained, the system is at the stability boundary, as shown in Fig. 7(b). Further, it may diverge to infinite magnitude corresponding to an unstable operation, as shown in Fig. 7(c). The prediction of the stability boundaries using the linearized fluid-flow model has been studied in [Chen et al., 2007].

From $n s-2$ simulations, however, the system is always under excitations from the TCP sources. The characteristic oscillation is embedded in other

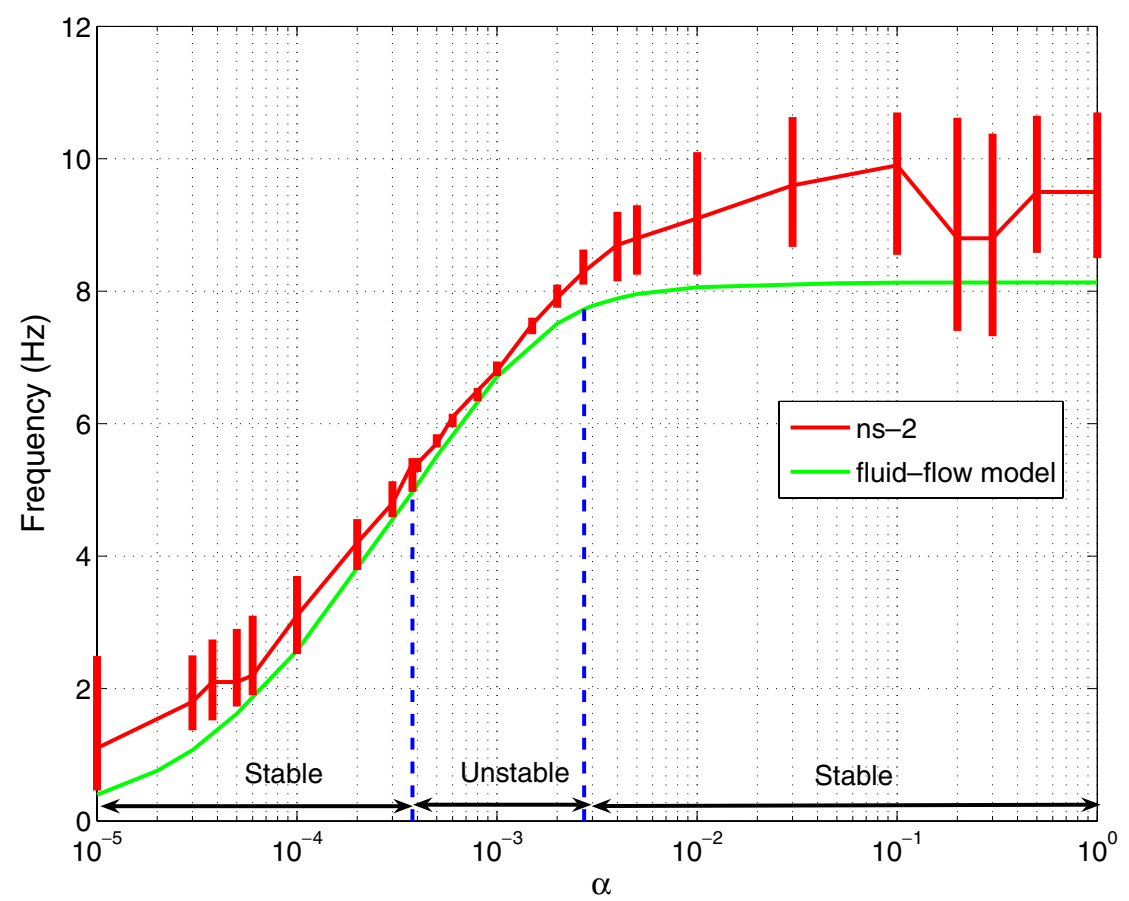

Fig. 6. Comparison of characteristic frequency $f_{c}$ from linearized fluid-flow model and peak oscillation frequency from $n s-2$ simulations. Bandwidths are indicated as vertical bars for the $n s$-2 data. System parameters are as listed in Table 1. 
Table 1. Parameters for $n s-2$ simulations.

\begin{tabular}{cc}
\hline$n s$-2 Parameters & Values \\
\hline$N$ & 170 \\
$\frac{C}{N}$ & $1.5 \mathrm{Mbps}$ \\
$R_{O}$ & $12 \mathrm{~ms}$ \\
$r_{o}$ & $19.46 \mathrm{~ms}$ \\
$X_{\max }$ & 512 packets \\
$q_{0}$ & 384 packets \\
$X_{\min }$ & 256 packets \\
Bottleneck delay & 6 ms \\
RED redwait_ & false \\
TCP ecn_- & 1 \\
\hline
\end{tabular}

random frequencies arising from the TCP sources. For instance, the fast Fourier transform of the waveforms in Fig. 1(a) has a frequency distribution shown in Fig. 8. The bandwidth of the peak frequency narrows down when the system enters instability, as shown in Fig. 9 for $\alpha=0.001$. The peak frequency and bandwidth of the distribution have been plotted in Fig. 6 for comparison with the characteristic frequency found from the linearized fluid-flow model. It can be observed that the peak oscillation frequency obtained from $n s-2$ simulations matches very well with the characteristic frequency obtained from the linearized fluid-flow model. The agreement is particularly good when the system enters into the instability boundary, implying that the system is dominated by the characteristic frequency.

The ideal TCP source window frequency shown in Fig. 3 is independent of $\alpha$ and is equal to a fixed value of $11.41 \mathrm{~Hz}$. As shown in Fig. 6, the characteristic frequency of the TCP-RED system is always lower than the ideal TCP source window frequency, meaning that the system encourages the TCP sources to send more packets. This encouragement intensifies as the damping factor of the system decreases and eventually reaches its lowest value in the middle of the unstable range, as shown in Fig. 6 .

Basically, the instantaneous queue size is proportional to the rate of arrival of the $N$ aggregated TCP source windows. When adding all the $N$

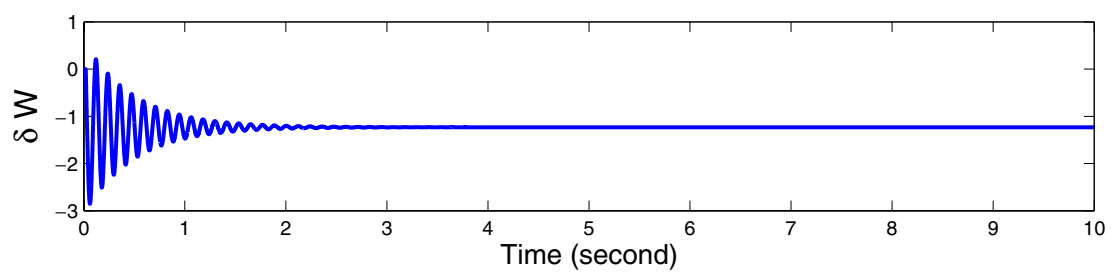

(a)

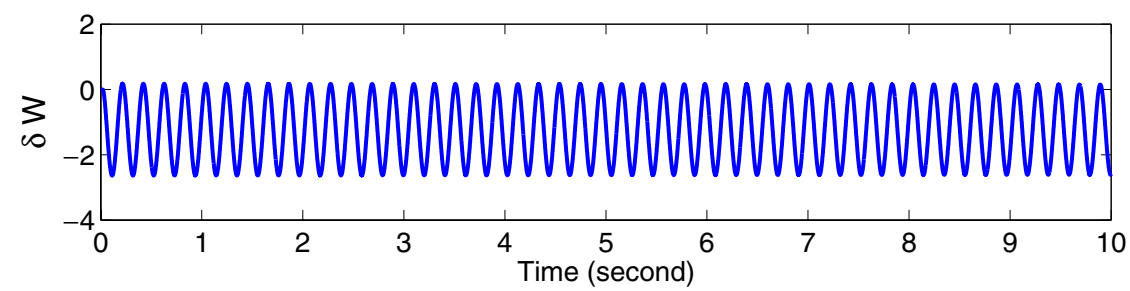

(b)

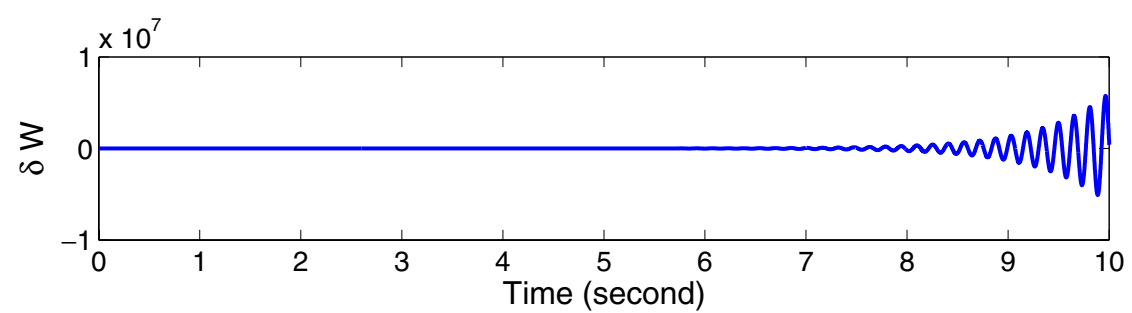

(c)

Fig. 7. Waveforms of perturbed window size found from linearized fluid-flow model for system parameters shown in Table 1. 


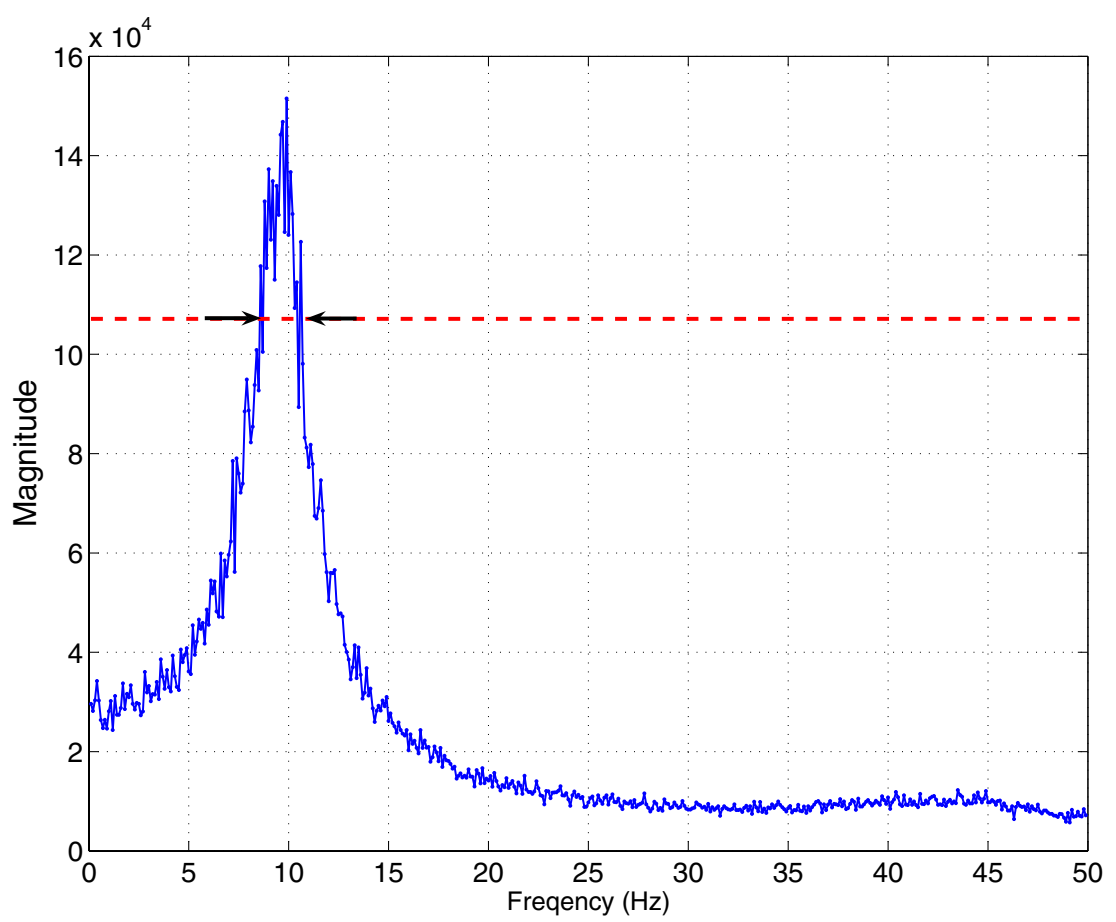

Fig. 8. Frequency distribution from FFT of the $n s-2$ simulated queue length waveform of Fig. 1(a). The distance between the two arrows is the bandwidth at this peak frequency.

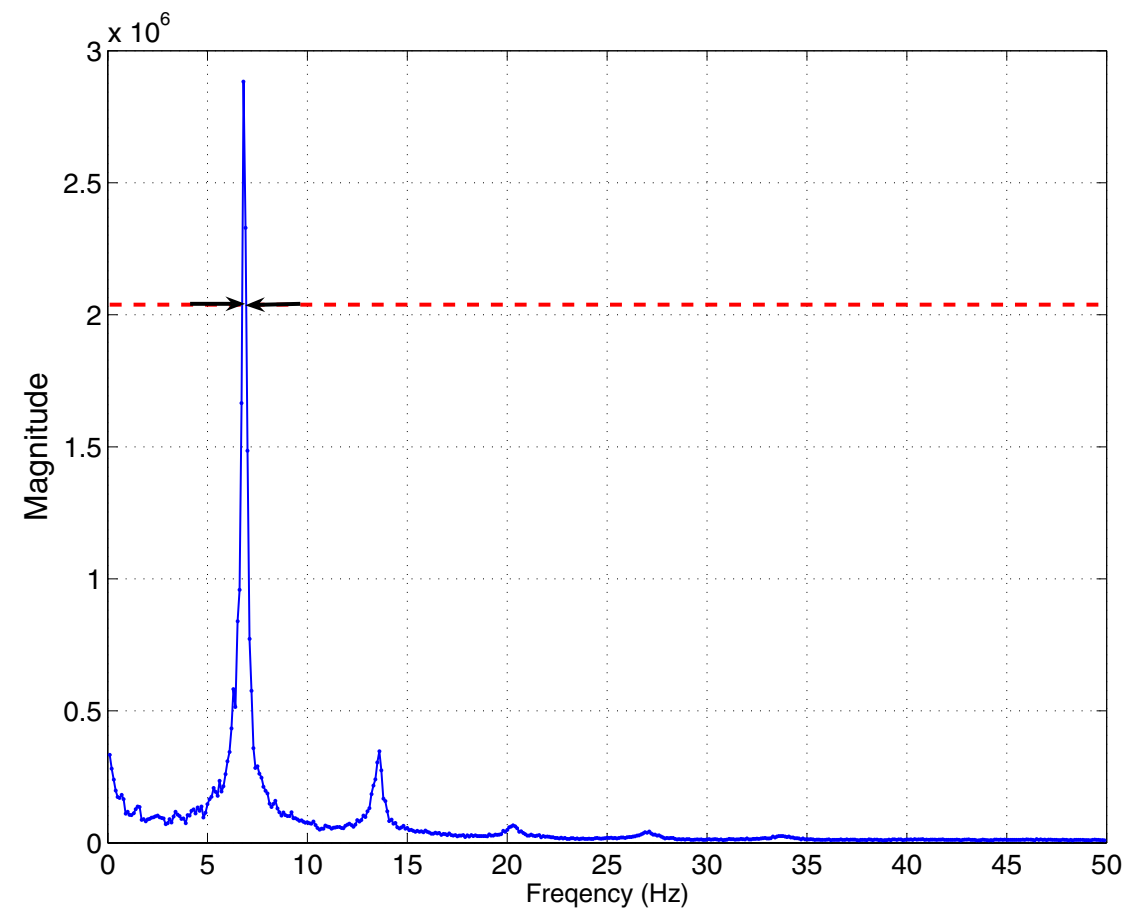

Fig. 9. Frequency distribution from FFT of the $n s-2$ simulated queue length waveform for $\alpha=0.001$. The distance between the two arrows is the bandwidth at this peak frequency. 
TCP flows together to get the instantaneous queue length, some dynamics specific to the TCP window algorithm will be lost. In the next subsection, we will study the distributions of individual window dynamics for the TCP source windows.

\subsection{Characteristic frequency and period doubling from a statistical perspective}

We consider the distribution of the TCP source window frequency, i.e. $1 / T$ (see (17) for the definition of $T$ ), for the aggregated source of $170 \mathrm{TCP}$ sources as well as an individual sending source selected from the 170 sources. Figures 10 and 11 show the distributions of the TCP source window frequency of the 52nd flow at $\alpha=0.1$ and of the 153rd flow at $\alpha=0.001$, both obtained from $n s-2$ simulations. Comparing the distributions of individual sources with that of the aggregated source, they all show similar distributions representing a fair share of bandwidth among the 170 TCP sources for a given $\alpha$. For instance, the distributions of Figs. 10 and 11 are similar to those in Figs. 12 and 15, respectively.

To reveal the dynamical behavior as the system goes across the stability boundary, we examine the distributions of the TCP window frequency on the two sides of the boundary, i.e. across the stable and unstable regions as shown in Fig. 6. Figures 12-21 show a sequence of the distributions of the window frequency from $n s$ - 2 simulations for $\alpha=0.1$, $\alpha=0.002, \alpha=0.0015, \alpha=0.001, \alpha=0.0008$, $\alpha=0.0006, \alpha=0.0005, \alpha=0.0004, \alpha=0.0001$ and $\alpha=0.00001$. We use a frequency resolution of $(2 / 375) \mathrm{Hz}$ to identify the peak frequency, and a frequency resolution of $1.6 \mathrm{~Hz}$ to identify the density which is shown in the small graph in each figure. The system exhibits a generally "smooth" distribution of the window frequency, in the sense that the distribution does not contain any spike at any frequency value. As $\alpha$ decreases and enters the unstable region as indicated from Fig. 6, the frequency distribution manifests period doubling which can be seen from the emergence of a small "bump" at half the characteristic frequency, as shown in Figs. 13-17. As $\alpha$ reduces further, period doubling subsides, as shown in Figs. 18-21. Also, a spike at $1 \mathrm{~Hz}$ corresponding to time-out saturation is clearly evident for the unstable (period-doubled) cases.

We should emphasize that due to the random nature of the TCP sources, the foregoing period doubling phenomenon can only be examined from a statistical perspective rather than in a strict

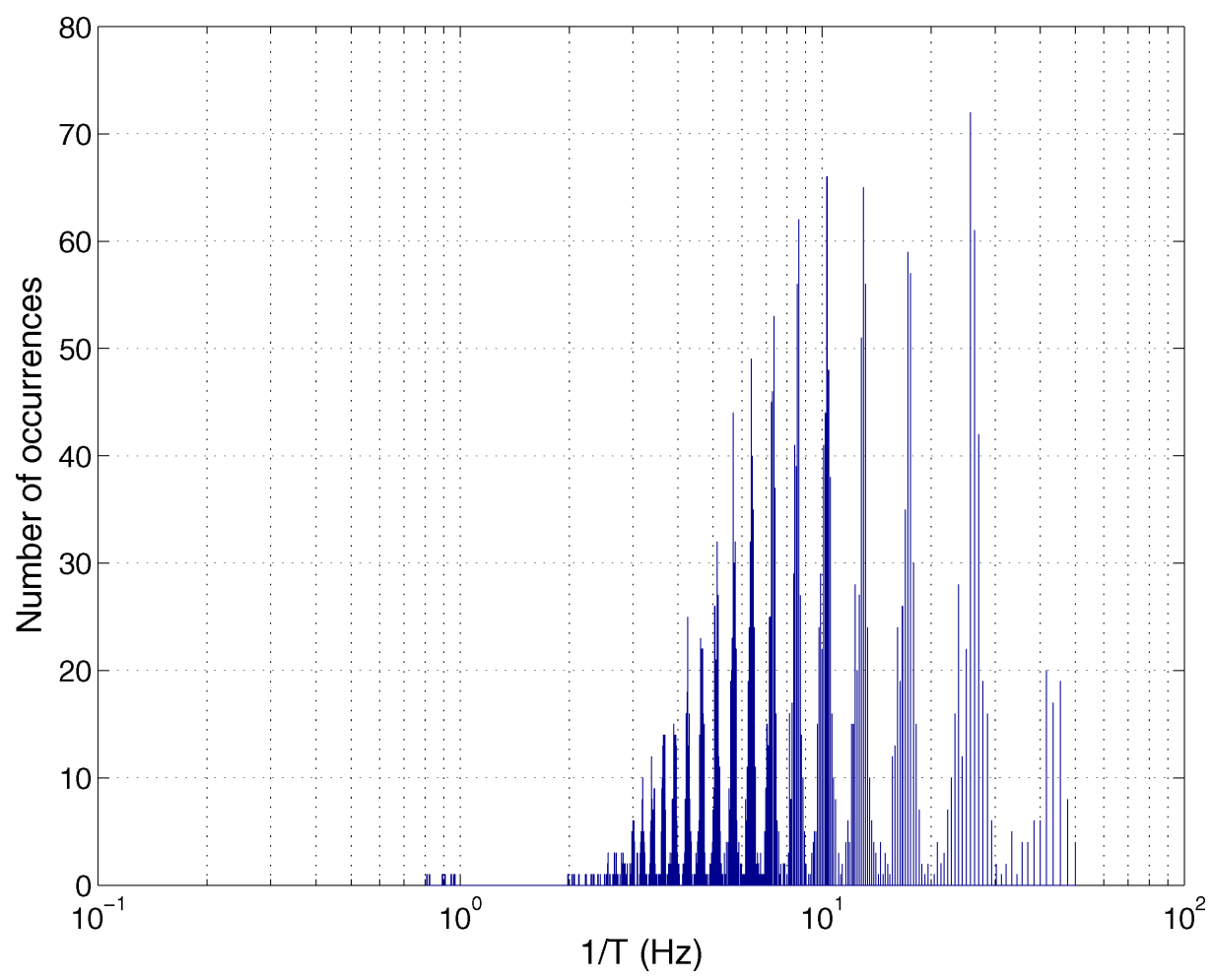

Fig. 10. Distribution of TCP source window frequency for the 52nd flow at $\alpha=0.1$. 


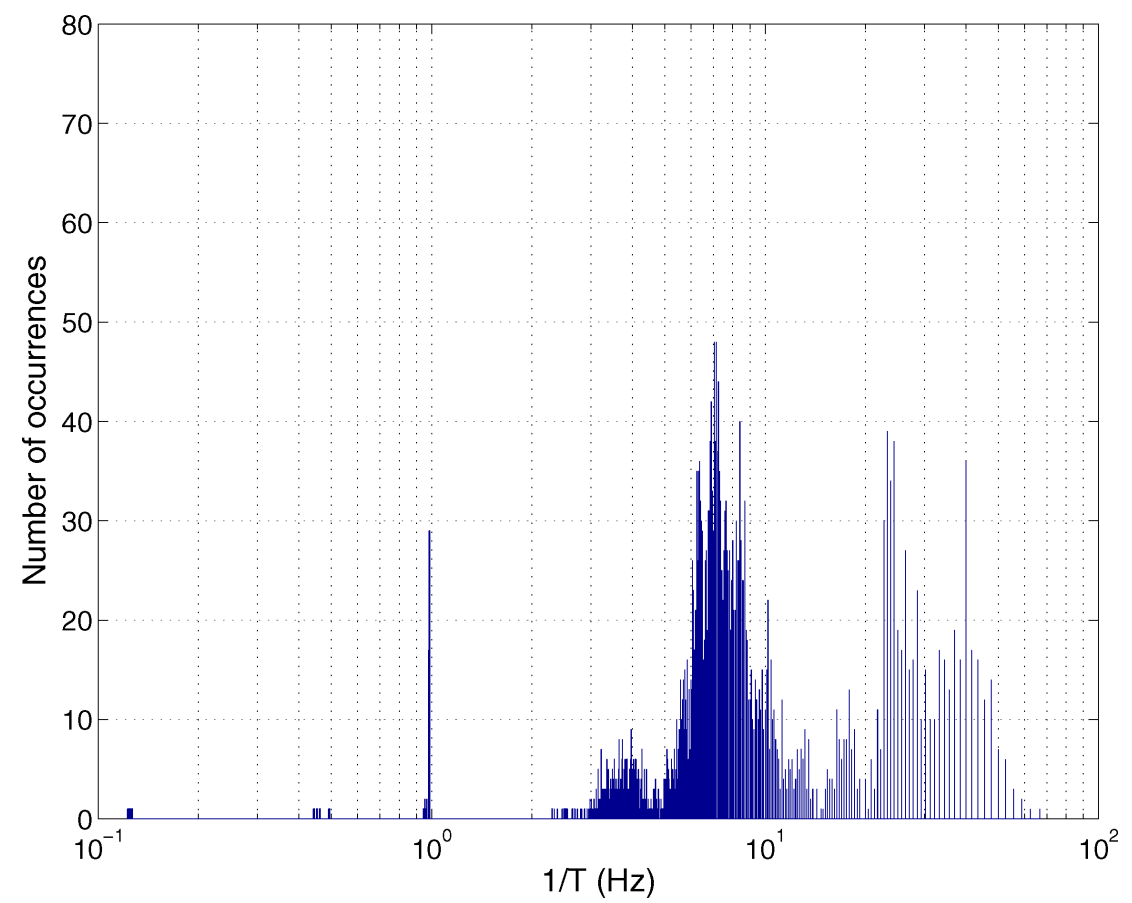

Fig. 11. Distribution of TCP source window frequency for the 153rd flow at $\alpha=0.001$. Period doubling in the statistical sense is clearly evident from the emergence of a small "bump" at half of the characteristic frequency.



Fig. 12. Distribution of TCP source window frequency for $\alpha=0.1$. 


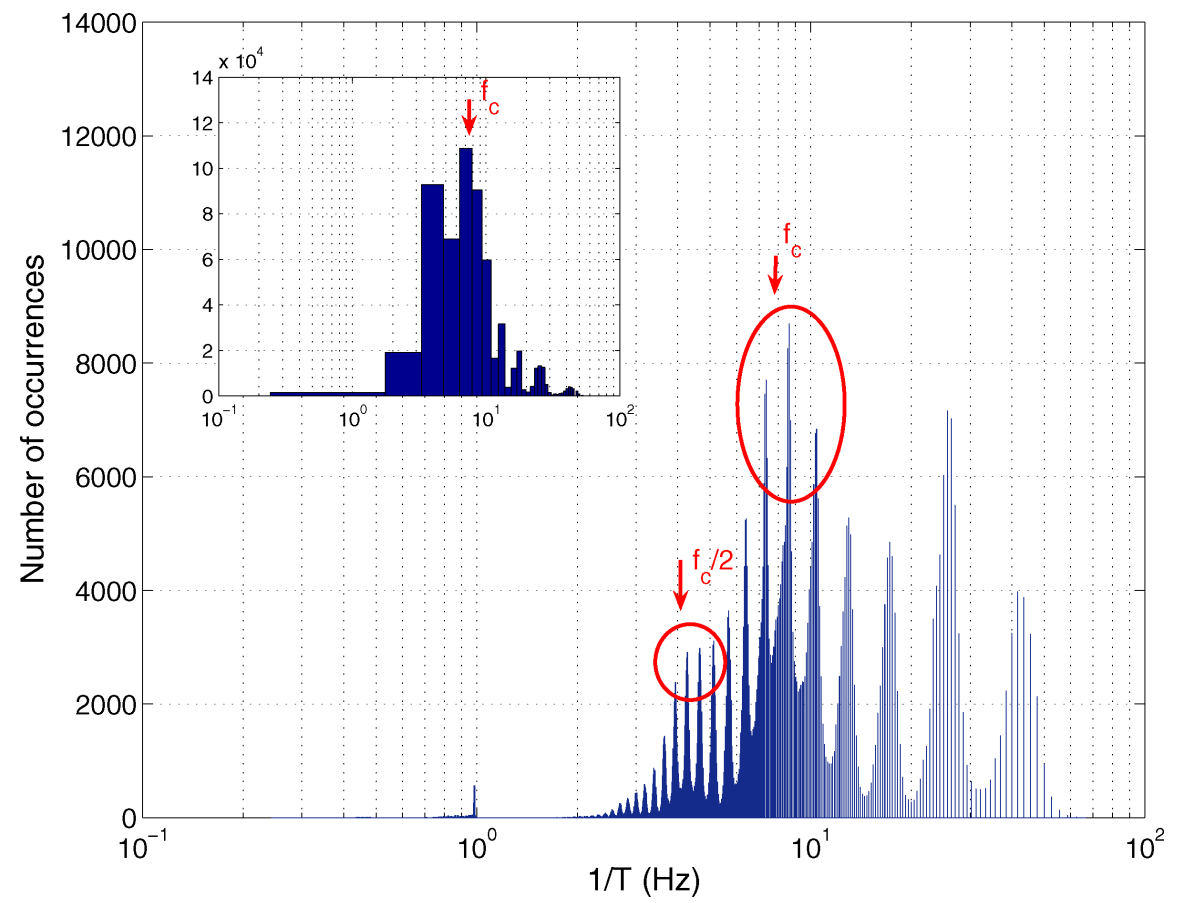

Fig. 13. Distribution of TCP source window frequency for $\alpha=0.002$. Period doubling in the statistical sense is clearly evident from the emergence of a small "bump" at half of the characteristic frequency. Impulse at $1 \mathrm{~Hz}$ reflects time-out saturation.

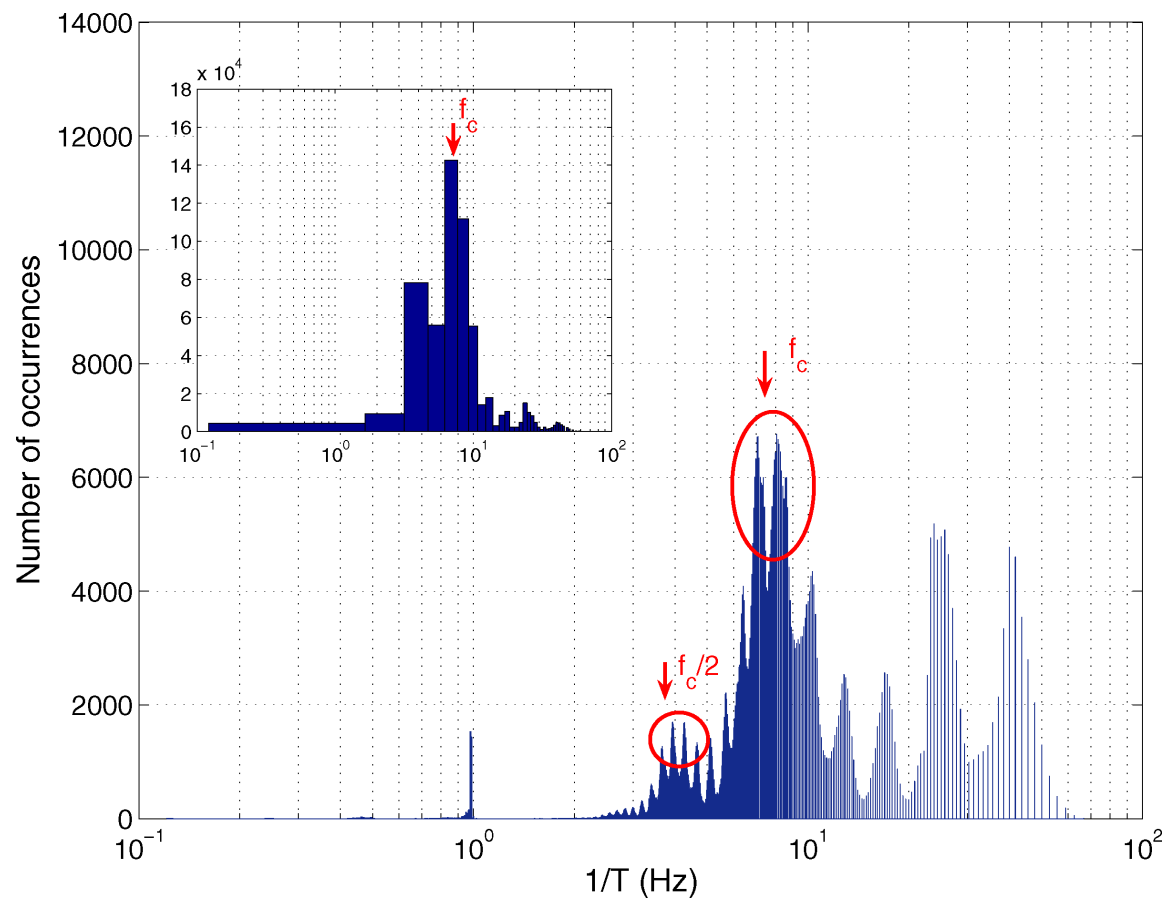

Fig. 14. Distribution of TCP source window frequency for $\alpha=0.0015$. Period doubling becomes more evident as the "bump" at half of the characteristic frequency grows. Impulse at $1 \mathrm{~Hz}$ reflects time-out saturation. 


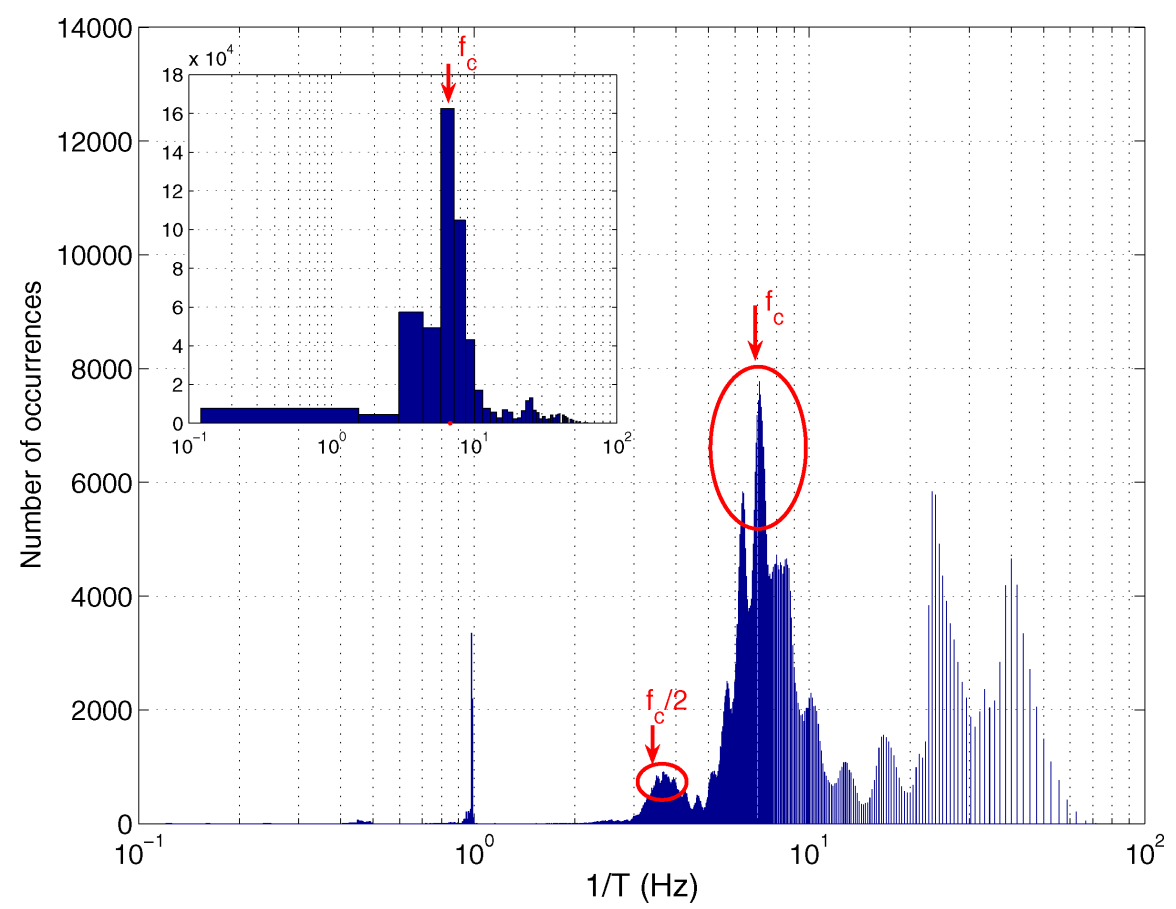

Fig. 15. Distribution of TCP source window frequency for $\alpha=0.001$. Period doubling persists as the "bump" at half of the characteristic frequency stays in the distribution. Impulse at $1 \mathrm{~Hz}$ reflects time-out saturation.

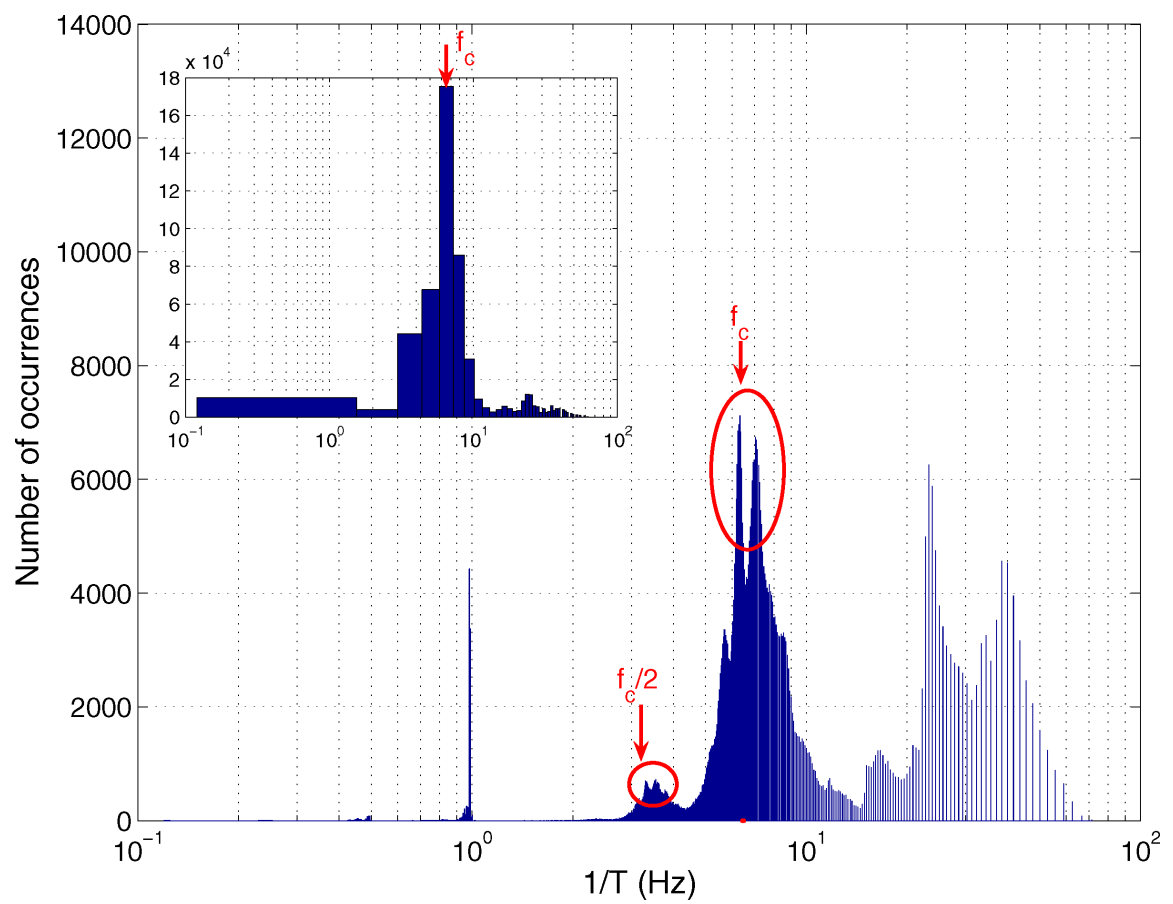

Fig. 16. Distribution of TCP source window frequency for $\alpha=0.0008$. Period doubling becomes less persistent as the "bump" at half of the characteristic frequency begins to shrink. Impulse at $1 \mathrm{~Hz}$ reflects time-out saturation. 




Fig. 17. Distribution of TCP source window frequency for $\alpha=0.0006$. Period doubling begins to subside. Impulse at $1 \mathrm{~Hz}$ reflects time-out saturation.

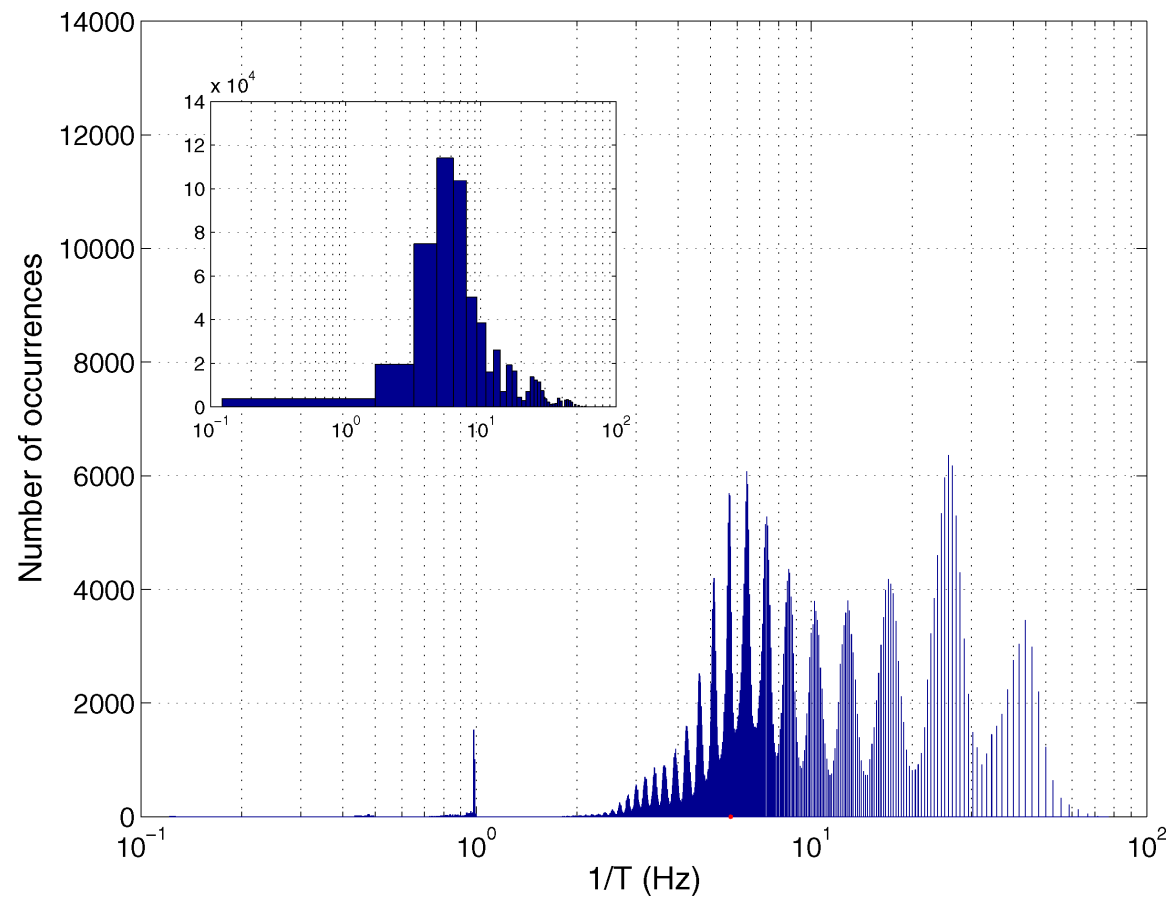

Fig. 18. Distribution of TCP source window frequency for $\alpha=0.0005$. Stability is about to resume as period doubling subsides. 


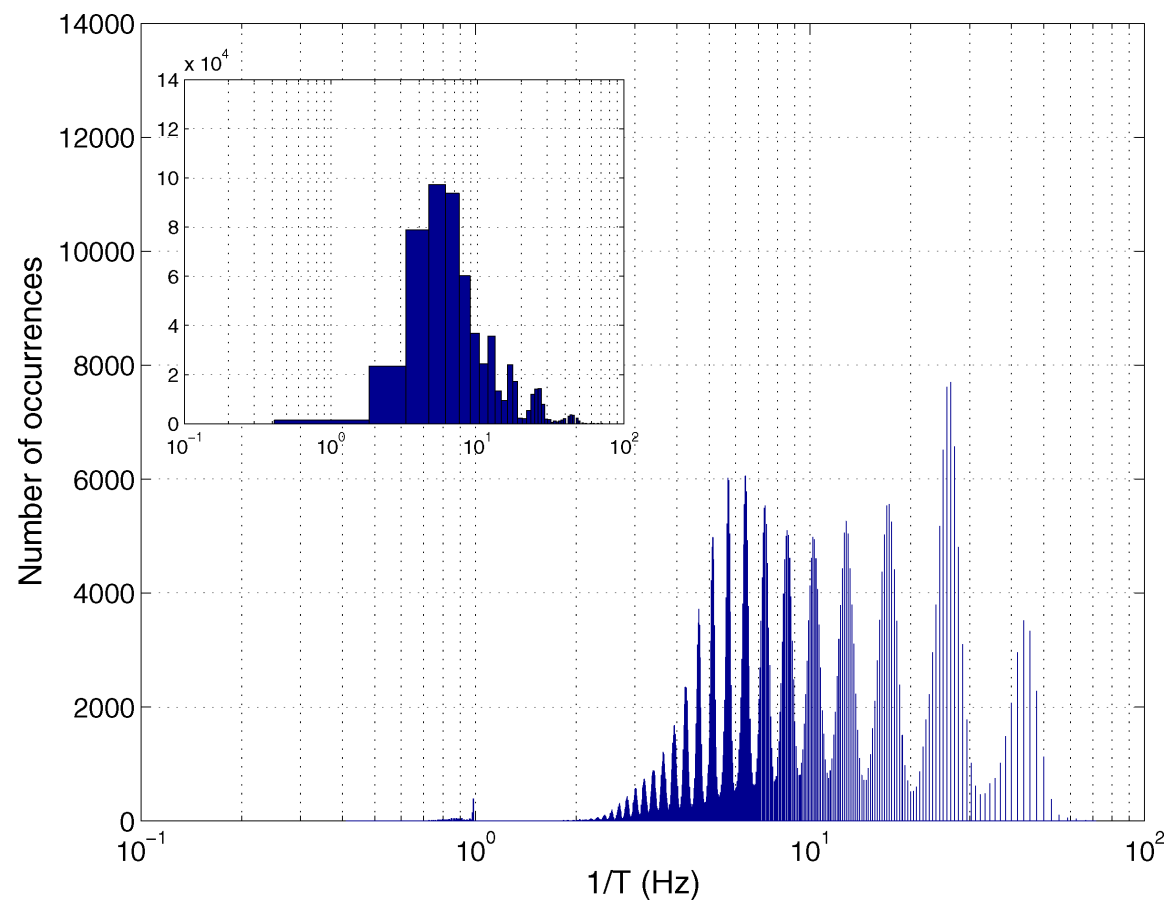

Fig. 19. Distribution of TCP source window frequency for $\alpha=0.0004$. Stability is resumed.



Fig. 20. Distribution of TCP source window frequency for $\alpha=0.0001$. 


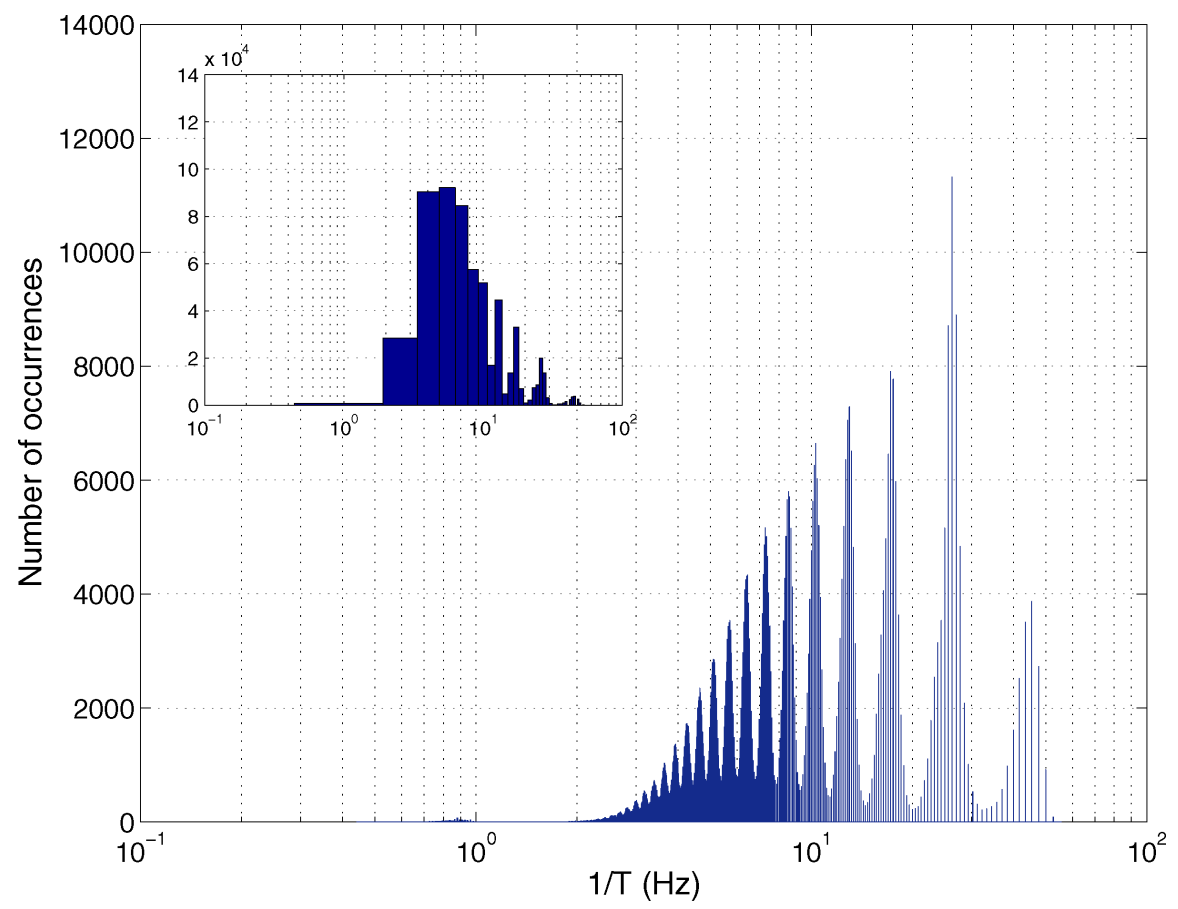

Fig. 21. Distribution of TCP source window frequency for $\alpha=0.00001$.

deterministic sense. The period doubling found here can be considered a true phenomenon because it is $n s-2$ verifiable. ${ }^{2}$

\subsection{Mechanism of oscillation}

The system manifests oscillatory behavior when it becomes unstable, and the frequency of oscillation synchronizes with the system's characteristic frequency which has been found analytically in this paper. At the same time, we observe period doubling in the sense that the distribution of the window frequency contains a significant component at half the characteristic frequency.

Detailed waveforms of TCP source window size have been shown in Figs. 4 and 5 for stable and unstable operations, respectively. The horizontal line indicates the average window size. Small circles are used to mark the windows whose frequencies fall between $f_{c} \pm 2 \mathrm{~Hz}$.

The TCP source window period is given by (17) and indicated in Fig. 3. When its distribution is considered, the period fluctuates as evidenced from Figs. 4 and 5. The averaged period is still given by (17). As shown from the small cycles in Figs. 4 and 5 , the TCP source window frequency follows the characteristic frequency of the system. Also, it can be observed that the TCP source window frequency synchronizes strongly with the system's characteristic frequency, as shown in Fig. 5. Such synchronization encourages a high flow rate (much higher than the average flow rate) that saturates the queue of the RED gateway. Thus, more and less ECNs at the two ends of the queue would saturate at $X_{\max }$ and $X_{\min }$ respectively. The number of time-outs, i.e. the peak of $1 \mathrm{~Hz}$, also increases dramatically.

When the system is stable, the RED gateway issues ECN markings periodically according to the TCP period. To have a fair distribution, each TCP source should receive randomly and an average of one ECN within the TCP period. This explains the smooth distribution of TCP source window frequency, in the sense that the distribution does not contain any spike at any frequency value, as shown in Figs. 12, 20 and 21. However, when the system becomes unstable, the population of TCP sources receiving ECNs at the characteristic period increases. The characteristic frequency recorded by $n s-2$ and shown in Fig. 6 is thus lower than the TCP steady-state window frequency which is $11.4 \mathrm{~Hz}$ (as given by (17)), resulting in a higher than average flow rate which must be reduced in some duration by issuing more ECNs from the RED

\footnotetext{
${ }^{2}$ In fact, many bifurcation phenomena found from some analytical models previously were not consistent with $n s$ - 2 simulations and hence remained as hypothetical phenomena which do not occur in reality.
} 
gateway. The short-term increase in ECNs is not necessarily a fair process which makes some TCP sources receive more than one ECN and some TCP sources miss a ECN within one characteristic period, resulting in an emergence of period doubling.

\section{Conclusion}

The fluid-flow model has been used to calculate the characteristic frequency of the TCP-RED system with multiple identical Reno TCP connections. The characteristic frequency has been compared with that obtained statistically from $n s-2$ simulations. Period doubling has been observed in a statistical sense from the $n s$ - 2 simulations using statistical frequency distribution of TCP source windows. Physical mechanism of onset of period doubling has been explained in terms of the difference in the TCP sending frequency and the system's characteristic frequency.

It should be emphasized that the main novelty in this paper is the provision of viable verifications using the industry standard $n s$ - 2 simulation tool. The bifurcation and stability results reported in this paper therefore reflect the true behavior of the actual system. This differs from most previous studies which derived their findings from analytical models, but were not verifiable by $n s$ - 2 simulations and could be inconsistent with the real TCP-RED system.

\section{References}

Brakmo, L. S. \& Peterson, L. L. [1995] "TCP Vegas: End-to-end congestion avoidance on a global Internet," IEEE J. Selected Areas in Comm. 13, 14651480.

Chen, L., Wang, X. F. \& Han, Z. Z. [2004] "Controlling bifurcation and chaos in Internet congestion control model," Int. J. Bifurcation and Chaos 14, 1863-1876.

Chen, X., Wong, S. C., Tse, C. K. \& Trajković, L. [2007] "Stability analysis of RED gateway with multiple TCP Reno connections," Proc. IEEE Int. Symp. Circuits and Systems, pp. 1429-1432.
Defense Advanced Research Projects Agency. [2007] "The network simulator — ns-2," [Online] http:// www.isi.edu/nsnam/ns/.

Firoiu, V. \& Borden, M. [2000] "A study of active queue management for congestion control," Proc. IEEE Infocom. 3, 1435-1444.

Floyd, S. \& Jacobson, V. [1997] "Random early detection gateways for congestion avoidance," IEEE/ACM Trans. Networking 1, 397-423.

Floyd, S., Gummadi, R. \& Shenker, S. [2001] "Adaptive RED: An algorithm for increasing the robustness of RED's active queue management," [Online] http://www.icir.org/floyd/red.html.

Hollot, C. V. \& Chait, Y. [2001] "Nonlinear stability analysis for a class of TCP/AQM networks," Proc. IEEE Conf. Decision and Control, pp. 2309-2314.

Hollot, C. V., Misra, V., Towsley, D. \& Gong, E. B. [2001] "A control theoretic analysis of RED," Proc. IEEE Infocom. 2, 1510-1519.

Jacobson, V. [1988] "Congestion avoidance and control," Proc. Symp. Communications Architectures and Protocol, pp. 314-329.

Jacobson, V. [1990] "Modified TCP congestion avoidance algorithm," [Online] ftp://ftp.ee.lbl.gov/email/ vanj.90apr30.txt.

Mathis, M., Semke, J., Mahdavi, J. \& Ott, T. [1997] "The macroscopic behavior of the TCP congestion avoidance algorithm," ACM SIGCOMM Comput. Commun. Rev. 27, 67-82.

Misra, V., Gong, W. B. \& Towsley, D. [2000] "Fluidbased analysis of a network of AQM routers supporting TCP flows with an application to RED," $A C M$ SIGCOMM Comput. Commun. Rev. 30, 151-160.

Park, E. C., Lim, H., Park, K. J. \& Choi, C. H. [2004] "Analysis and design of the virtual rate control algorithm for stabilizing queues in TCP networks," Comput. Networks 44, 17-41.

Ranjan, P. \& Abed, E. H. [2002] "Nonlinear instabilities in TCP-RED," Proc. IEEE Infocom. 1, 249-258.

Wong, S. C., Tse, C. K. \& Lau, F. C. M. [2005] "Characteristic oscillation and intermittency in TCP-RED gateway," Proc. Int. Symp. Nonlinear Theory and Its Applications, pp. 90-93. 